\title{
Two Algorithms for Weight Problems
}

\section{Chun Shi ${ }^{1, a}$, Xin Yin ${ }^{1}$, Chunyu $\mathrm{Li}^{1, \mathrm{~b}}$, Ruyin $\mathrm{Xu}^{1}$ and Shuqian $\mathrm{He}^{1}$}

${ }^{1}$ Lab. Digital Medium, School of Information Science \& Technology, Hainan Normal University, Haikou, Hainan 571127, China

abyshichun@hainnu.edu.cn, ${ }^{b}$ lichunyu_hn@126.com

Keywords: Weight problem; Greedy algorithm; Dynamic programming; C++

\begin{abstract}
Research on weight problem is helpful to solve other problems, and it has practical significance for the design of the algorithm and the solution of the real life. The purpose of this paper is to introduce the solution for the weight problem with both greedy algorithm and dynamic programming. We mainly discuss the difference between these two algorithms based on different solution methods. The algorithms in this paper are described in $\mathrm{C}$ language, natural language or charts.
\end{abstract}

\section{浅谈砝码称重问题的两种算法}

石春 $^{1, a}$, 尹 鍂 ${ }^{1}$ ，李春雨 ${ }^{1, b}$, 许茹茵 ${ }^{1}$ ，何书前 ${ }^{1}$

1. 海南师范大学 信息科学技术学院, 海南 海口 571127

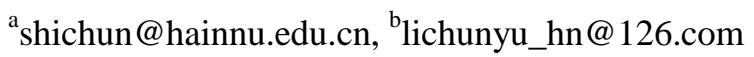

摘要: 砝码称重问题的研究有助于解决其它各种拓展出的问题, 对算法设计和实际生活中问题 的解决都具有现实的意义。对砝码称重问题, 用贪心算法与动态规划两种方法求解。从求解 思路介绍两种算法的差异。算法使用 C 语言, 自然语言以及图表描述。

关键词: 称重问题; 贪心算法; 动态规划； $\mathrm{C}++$

\section{1. 引言}

在算法学习和研究过程中, 针对同一个问题, 采用不同的算法进行分析, 可以有效加深对算 法特征的理解，同时也为解决其他问题开拓了更广阔的思路 [1] [2]。

砝码称重是算法分析中一个经典的问题, 已经有学者从多个方面进行了探讨 [2] [3] [4], 本文 尝试从贪心算法 [5] [6]和动态规划 [7] [8]给出算法解决方案之间的差异。

\section{2. 砝码称重的问题描述}

设有 $1 \mathrm{~g}, 2 \mathrm{~g}, 5 \mathrm{~g}$ 的砝码各若干枚。试设计算法, 计算可用这些砝码称出多少种不同的重量。 例如, 有 $1 \mathrm{~g}, 2 \mathrm{~g}, 5 \mathrm{~g}$ 的砝码各 1 枚, 则可用它们称出 $1 \mathrm{~g}, 2 \mathrm{~g}, 3 \mathrm{~g}, 5 \mathrm{~g}, 6 \mathrm{~g}, 7 \mathrm{~g}, 8 \mathrm{~g}$ 共 7 种 不同的重量。不能由砝码组合称出 $4 \mathrm{~g}$ 的重量。

当砝码的数量不一致, 或者部分砝码 (比如, $1 \mathrm{~g}$ 砝码) 没有的时候, 组合的砝码所能称出的 重量会更少。从数值关系进行分析, 砝码组合所称出不同重量的数量不会超过砝码的总重量 之和。即, 砝码总重量的数值, 也就是组合砝码所能称出不同重量的最大数量。直观理解, 砝码不能称出超过总重量的重量。

针对砝码称重问题, 下面分别从动态规划算法和贪心算法给出算法分析比较。 


\section{3. 算法分析}

\section{1. 动态规划算法分析}

动态规划算法的基本思想是将待求解问题分解成若干子问题，按顺序求解子问题，一般一个 子问题的解会用到前一个子问题的解，最后从这些子问题的解得到原问题的解。

砝码称重问题是算法分析中的常规问题, 通常建议用动态规划算法进行分析解决 [王晓东一算 法设计与分析。因为在对砝码称重进行分析的时候，存在多个砝码的重量被重复叠加计算的 情况, 比如, $3 g=2 g+1 g$ 和 $8 g=5 g+2 g+1 g$ 中, $(1 g+2 g)$ 被重复计算了两次。这说明, 在计算 重量的过程中, 每次单独计算砝码重量的事件之间存在一定的关联性, 即, 部分砝码叠加计 算事件之间并不是独立的。当砝码数量足够多的时候，这种砝码叠加计算的重复性会更多。 这满足动态规划算法中的子问题 (部分砝码叠加计算事件) 重叠性质。很显然, 当去掉若干 个砝码得到的子问题, 其组合称重范围不会超出原砝码数量, 即, 满足最优子结构性质。因 此，采用动态规划算法计算砝码称重问题是可以得到最优解。

\section{2. 贪心算法分析}

贪心算法 (又称贪婪算法) 是指, 在对问题求解时, 总是做出在当前看来是最好的选择。也 就是说，不从整体最优上加以考虑，他所做出的仅是在某种意义上的局部最优解。

砝码称重问题类似超市找零问题: 当我们在超市购物需要找零时, 收银员总是先拿找零范围 内面值最大的人民币若干，然后再拿面值次大的…… 直到拿够找零的钱。其贪心算法思路 为:在不超过找零金额的条件下, 每次尽可能挑选面值最大的人民币, 在金额不足的情况下, 再 采用同样的方法考虑面值次大的人民币补足剩余所需金额, 依此类推, 直到找足所需金额为 止。

当砝码称重问题满足贪心选择性质的时候, 可以采用类似的求解思路。所谓贪心选择性质是 指所求问题的整体最优解可以通过一系列局部最优的选择，即贪心选择来达到。

在上述砝码称重问题中，可以对质量范围类的每一种质量用贪心算法判断能否称出，最后统 计能称出的质量的个数即为砝码称重问题的解。但是砝码质量的求解并不总是满足贪心选择 性质。比如, $1 \mathrm{~g}, 2 \mathrm{~g}, 5 \mathrm{~g}$ 的砝码个数分别为 $(0,3,1)$ 时称 $6 \mathrm{~g}$ 的质量。采用贪心算法进行 求解, 会首先 选择 $5 \mathrm{~g}$ 的砝码, 而选择了 $5 \mathrm{~g}$ 的砝码是不可行的, 所以贪心算法这种情况是称 不出 $6 \mathrm{~g}$ 的。因此砝码称重问题用贪心算法求解存在问题, 对于原问题并不是每次都能得到正 确解。

因此, 要用贪心算法解决砝码称重问题, 需要对输入数据进行分析。若输入数据满足贪心选 择性质，则可直接用贪心算法求解，若不满足，则另外求解。

那么，什么样的输入数据才能满足贪心选择性质呢？我们做如下一个假设。

假设质量为 $1 \mathrm{~g}$ 的砝码个数不为 0 , 则满足贪心选择性质。

证明：设 $1 g 、 2 g 、 5 g$ 的砝码个数为 $(a 、 b 、 c)$ ( $a 、 b 、 c$ 都为正整数)。质量 $M(0<M<=a+2 b+5 c)$ 能够被称出 (只用考虑能够称出的质量, 不能称出的质量满不满足贪心选择都不能称出, 所 以不予考虑）, $M$ 经过若干次贪心选择后质量为 $m$, 此时 $m$ 大于剩余砝码中最大砝码的质量小 于 2 倍最大砝码质量。当不满足贪心选择性质的情况时, 那么 $\mathrm{m}$ 只能由小的砝码称出, 选择 质量大的砝码后不能称出。例如 $m=6$ 时, 剩余砝码个数为 $(0 、 3 、 1) 。 6 g$ 能够由 0 个 $1 \mathrm{~g}$ 和 3 个 $2 \mathrm{~g}$ 称出, 即 $6=0 * 1+2 * 3$ 。但选择了 $5 \mathrm{~g}$ 的砝码则称不出, 即 $6-5=1$ 不能由剩下的砝码称出。 若要使输入数据满足贪心选择性质。质量 $M$ 经过一系列的贪心选择后剩余质量为 $\mathrm{m}$, 此时 $5 \mathrm{~g}$ 的砝码还有剩余, 且 $\mathrm{m}=\mathrm{a}+2 \mathrm{~b}$ 。根据贪心算法的性质会继续选择 $5 \mathrm{~g}$ 的砝码, 选择 $5 \mathrm{~g}$ 砝码后 质量为 $m-5$ 。若要使 $m-5$ 能够被称出, 则 $m-5=(a-5)+2 b ; m-5=(a-3)+2(b-1) ; m-5=(a-1)+2(b-2)$; 三个等式必须至少满足一个, 即 $(a-5)>=0$ 或 $(a-3)>=0$ 或 $(a-1)>=0$, 即 $a>=1$ 。所以 $a$ 不为 0 时满足贪心选择性质。

由以上证明可得出一个结论：只有当输入数据（a,b, c) $\mathrm{a} \neq 0$ 。即 $1 \mathrm{~g}$ 的砝码个数不为 0 时, 输入数据满足贪心选择。 


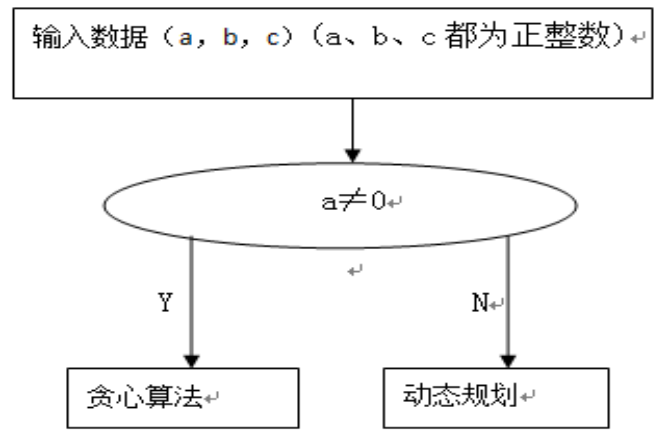

图 2-1 算法流程图

由以上算法分析可知, 砝码称重问题可以用动态规划算法求解, 也可使用贪心算法加动态规 划算法求解 (对输入数据分析, 若满足贪心选择性质则用贪心算法求解, 若不满足则用动态 规划算法求解 $)$

基于 $\mathrm{C}++$ 程序设计语言 [9] [10] 的动态规划算法程序实现:

Begin（算法开始）

输入 sum, tab $[0]=1$, number [], weight [] ;

for $(i=0 ; i<3 ; i++) / /$ 三种砝码

$\{$ for $(j=0 ; j<$ number $[i] ; j++) / /$ 每种砝码的个数

$\left\{\right.$ for $\left(z=\right.$ sum; $z>=$ weight $\left.[i] ; z^{--}\right) / /$sum 为可以称出的最大重量

$\left\{\right.$ if (tab $\left[z^{-}\right.$weight $\left.\left.[i]\right]==1\right) / /$ 判断质量是否可以被称出 $\operatorname{tab}[z]=1 ;\}\}\} / /$ 若能称出, 标记为 1

统计 $\mathrm{tab}[]$ 中 1 的个数将值赋给 $K$;

输出 $K$

End （算法结束）

满足贪心选择性质的输入，贪心算法程序实现：

Begin（算法开始）

输入 n, sum, number[], weight [] ;

For (i=sum; $i>0 ; i--)$

$\{$ for $(j=n ; j>=0 ; j--) / / n$ 为砝码总个数

$\{a=i-w e i g h t[j]$;

If $(a>0)$

则 $i=i-w e i g h t[j]$;

If $(a==0)$

则 $\{c=1 ;$ break; $\}$

If $(a<0)$

则 $a=i ;\}$

If $(\mathrm{c}==1)$

则 $\operatorname{tab}[i]=1 ;\}$

统计 $\operatorname{tab}[]$ 中 1 的个数 $K$;

输出 $K$

End （算法结束）

\section{4. 两种算法对比与总结}

动态规划算法解决砝码称重问题时主要是对砝码的处理 , 时间复杂度为 0 （n*sum）（n 为砝 
码个数, sum 为可以称出的最大质量, 即所有砝码质量和）。

贪心算法解决砝码称重问题时, 若输入数据满足贪心选择性质。则用贪心算法求解。对某一 质量用贪心算法思路判断是否能被称出, 其时间复杂度为 $0(\mathrm{n}) \quad \mathrm{n}$ 为砝码个数）。贪心就 是一种特殊的动态规划, 只不过它是能够 “按一定贪心选择的策略总是直接选择其中的一种 最优解 “的动态规划, 对于砝码称重问题, 若输入数据满足贪心选择性质就从普通的动态规 划变成贪心了, 计算量减少了, 时间成本自然也会降低了, 但是由于要对质量范围内的每一 种质量用贪心策略求解, 所以贪心算法解砝码称重问题时间复杂度为 0 ( $\mathrm{n} * \mathrm{sum}$ ) ( $\mathrm{n}$ 为砝码 个数, sum 为可以称出的最大质量, 即所有砝码质量和)

虽然两种算法的时间复杂度一样, 但是求解思路确实截然不同。通过对两种算法进行分析, 加深了对算法特征的理解，同时也为解决其他问题开拓了更广阔的思路。

\section{5. 致谢}

感谢国家自然科学基金项目 (No.61362016, 61502127, 61562022, 61562023), 海南省自然科学基金项目 (No. 20156225) 和海南省高等学校科研项目（No.Hnky2016-17, Hnky2015-24）支持。

\section{Acknowledgement}

This research was financially supported by NSFC (No.61362016, 61502127, 61562022, 61562023), NSF of Hainan Province (No.20156225) and Scientific Research Project of Hainan Province (No.Hnky2016-17, Hnky2015-24).

\section{Reference}

[1] Wang Xiaodong. Design and analysis of computer algorithms (Fourth Edition) [M]. Publishing House of electronics industry, 2007.

[2] XU Zhi-min. Analysis and Exploration of Various Algorithms of the Weight Problem. Journal of Hefei University, 21(1):44-48, 2011.

[3] Song Shibo, Li Yan, Huang Yonghui. Race weighing [J]. mathematical problem-solving research: high school edition, 2006 (12): 6-6.

[4] Chu Jinlin. determination of the state of dynamic programming problem [J]. Journal of Anhui Institute of Education, 2003, 21 (3): 11-14.

[5] Chang Youqu, Xiao Guiyuan, Zeng min. Research on the greedy algorithm [J]. Journal of Chongqing Electric Power College, 2008, 13 (3): 40-42.

[6] Xiao Heng. Analysis of the greedy algorithm [J]. office automation, 2009 (18): 25-26.

[7] Dong Junjun. Comparison and Analysis of Dynamic Programming Algorithm and Greedy Algorithm [J]. software guide, 2008 (2): 129-130.

[8] Zhang Aihua, Guo Xiyue, Chen Qianjun. The dynamic programming algorithm research and analysis [J]. software guide, 2014 (12).

[9] Pan Dafu, Wang Bo, Zhou Zhiqiang. Study of Matlab and C/C++ mixed programming technology [J]. computer engineering and design, 2009, 30 (2): 465-468.

[10]Li Juan, Zhang Qin. Visual C++ Hybrid programming based on Dynamic Link Library [J], computer engineering and design, 2010, 31 (9): 2140-2143.

作者简介: 石春 $(1977-)$, 男, 江西省鄱阳县, 副教授, 主要研究方向: 无线通信协议、 
信息处理和软件工程, E-mail: byshichun@hainnu. edu.cn。 